\title{
Mujeres rurales emprendedoras, detonadoras de desarrollo económico: binomio colaboración - empoderamiento
}

Entrepreneurial rural women, economic development detoners: binding collaboration - empowerment

Isabel Pérez Pérez

Docente/Investigadora

Titular de la Escuela de Ciencias Administrativas Istmo Costa, Campus IX, Tonalá. Universidad Autónoma de Chiapas. Investigadora Nacional Nivel 1 Investigadora Estatal Honoraria Nivel VI. Perfil Prodep. isabelperez1491@hotmail.com

Recepción: 05/01/2017. Aceptación: 21/02/2017. Publicación: 24/05/2018

Citación sugerida:

Pérez Pérez, I. (2018). Mujeres rurales emprendedoras, detonadoras de desarrollo económico: binomio colaboración - empoderamiento. 3C Empresa: investigación y pensamiento crítico, 7(2), 26-43. DOI: http://dx.doi. org/10.17993/3cemp.2018.070234.26-43/ 


\section{RESUMEN}

La mujer rural emprendedora es factor clave de la sociedad mexicana que genera recursos económicos a las familias. En Chiapas (México), no se pueden excluir de la situación específica de las mujeres rurales, las cuales representan el 50,4\% del total de las mujeres mexicanas (CEAMEG, 2014). La mayoría de este colectivo, están en situación de pobreza extrema. Además de vivir en comunidades rurales, con una inadecuada infraestructura de servicios, escasos servicios de salud o educativos, y con necesidades básicas insatisfechas en la mayoría de los hogares, viven dominadas por el sistema "machista" propia de una cultura rural, sin reconocimiento de sus derechos, ni de sus labores dentro y fuera del hogar.

Por ello, se presenta este estudio que se realizó con una metodología mixta, cualitativa y cuantitativa. La recolección de información utilizó los siguientes instrumentos metodológicos: un grupo focal y la aplicación de una encuesta semi estructurada a los participantes del mismo grupo focal (hombres, mujeres y jóvenes, todos ellos mayores de 18 años, vendedores de pescado en la periferia del mercado público Manuel Larrainzar). El resultado reflejó que de las 17 personas que comercializan su producto en este sitio, 15 son mujeres, y únicamente 2 hombres; de ellos, 10 son casados (as), 3 solteras, 1 que vive en unión libre, 1 madre soltera, 1 divorciada y 1 viuda, como se puede observar el rol protagónico de la mujer en esta actividad comercial es predominante.

\section{ABSTRACT}

Rural women entrepreneurs is key factor in Mexican society that generates income to families. In Chiapas (Mexico), may not be excluded from the specific situation of rural women, which account for 50.4 per cent of the total of Mexican women (CEAMEG, 2014). The majority of this group, are in extreme poverty. In addition to live in communities that are rural, with inadequate infrastructure services, scarce health services or educational, and basic needs unmet in most households, they live dominated by the "macho" system of a rural culture, without recognition of their rights, or their work inside and outside the home.

Why is this study which was carried out with a joint, qualitative and quantitative methodology. The collection of information used the following methodological instruments: implementation of a survey and a focus group semi structured to participants of the same target group (men, women and young people, all of them older than 18 years, fish sellers in the periphery of the public market Manuel Larrainzar). The result reflected that of the 17 people who marketyour product on this site, 15 are women, and only 2 men; of them, 10 are married (as), 3 single and 1 who lives in free union, 1 single mother, divorced 1 and 1 widow, as you can see the role of women in this commercial activity is predominant. 


\section{PALABRAS CLAVE}

Mujeres rurales, Mercados públicos, Empoderamiento, Economía.

\section{KEY WORDS}

Rural women, Public markets, Empowerment, Economy. 


\section{INTRODUCCIÓN}

Con este estudio se logró identificar la problemática que viven las mujeres emprendedoras rurales que vienen a la ciudad a buscar un sitio en los mercados locales para la venta de su producto (pescado) y tienen que ubicarse en la periferia del mercado público, puesto que no tienen un sitio definido para realizar la comercialización de su mercancía. Considerando que estos lugares ofrecen una serie de oportunidades para vendedores y compradores de distintos tipos de productos y servicios.

Por ello, primeramente se elaboró un censo que permitió obtener datos e información para posteriormente proponerles una forma de organización que les facilite realizar esta actividad de forma más segura y puedan obtener ganancias que les provea para que puedan vivir de una manera digna.

En segundo lugar, se ubicaron los problemas a que se enfrentan en el día a día, estas mujeres para realizar sus actividades diarias en una matriz FODA. Posteriormente, se les diseñaron documentos contables donde podrán llevar un registro que concentre todas sus operaciones diarias como son sus compras y ventas, así como lo que se invierte y cuanto se obtiene de ingreso económico al efectuar la comercialización de su producto, si se obtiene una ganancia o no.

Considerando que los mercados públicos proveen grandes oportunidades de negocio, para los que ofrecen productos o servicios por representar un sitio donde oferentes y demandantes convergen para buscar mejores oportunidades, tanto en los precios, como en las oportunidades de ventas comerciales.

\subsection{CARACTERÍSTICAS DE LA POBLACIÓN OBJETO DE ESTUDIO}

En las comunidades rurales, existen muy pocas actividades que pueda absorber la mano de obra femenina, lo que implica que las mujeres en edad productiva genere su propio empleo, por medio de la venta y comercialización de todos aquellos productos o servicios que impliquen una oportunidad de trabajo, que les provea ingresos mínimos necesarios para su subsistencia y la de su familia.

Un ejemplo, es el que realizan las mujeres rurales emprendedoras que venden pescado en la periferia del mercado público Manuel Larrainzar, ubicado en Tonalá, Chiapas. La importancia de esta actividad radica en la cadena de valor que genera este proceso de compra/venta para las comunidades, aun cuando no se considera un trabajo formal, es una ocupación digna. 
Observándose que la situación económica de estas personas, no está tan deprimida como en otras familias que no realizan actividades de comercialización y que dependen únicamente de los apoyos gubernamentales que mes a mes reciben. Este es un factor muy importante si se considera que los hijos, principalmente mujeres, forman parte de la fuerza de trabajo en esta actividad (Pérez, Orozco \& Cruz, 2014).

\section{La situación económica de las mujeres rurales que venden pescado no está tan deprimida como en otras familias que no realizan actividades de comercialización y que dependen de los apoyos gubernamentales.}

Según García (2003), el trabajo manual es significativo por el hecho de no contar con tecnología, estructura un proceso de trabajo diferente al de una organización industrial con producción en serie. La producción está orientada hasta ahora, a la generación de un ingreso familiar de subsistencia (ver figuras 1,2 y 3$)$.

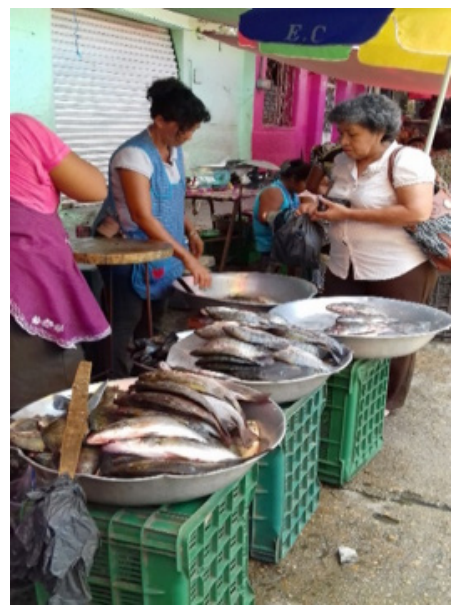

Figura 1. Mujeres rurales vendedoras de pescado en el mercado público Manuel Larrainzar en Tonalá, Chiapas. 


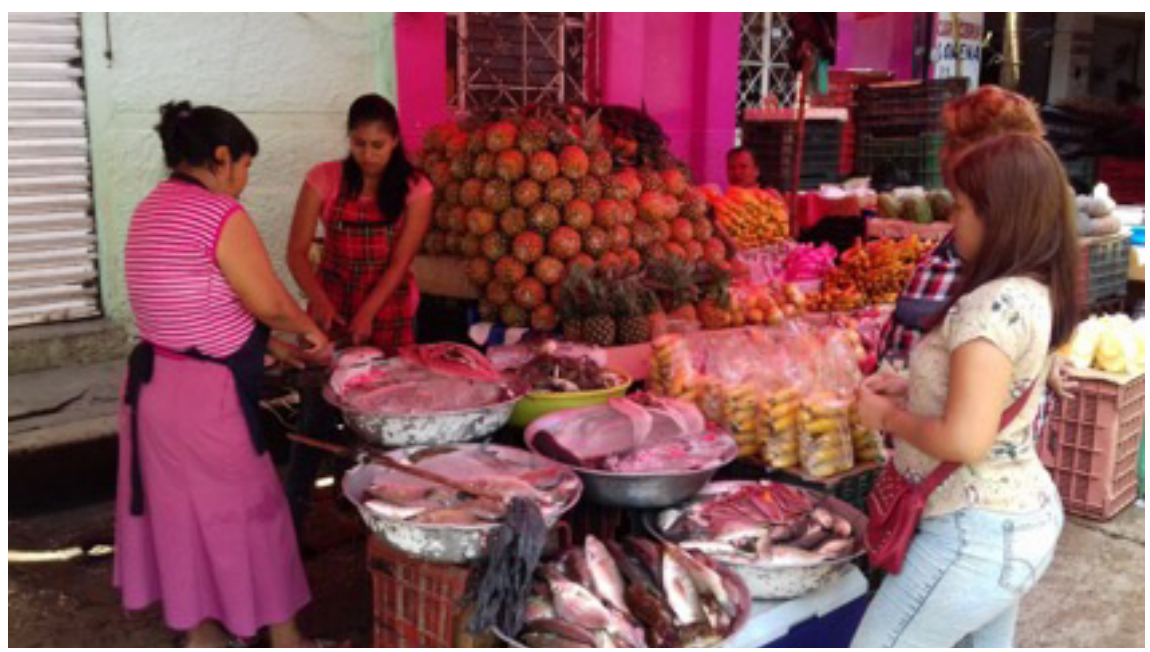

Figura 2. Mujeres rurales vendedoras de pescado en el mercado público Manuel Larrainzar en Tonalá, Chiapas.

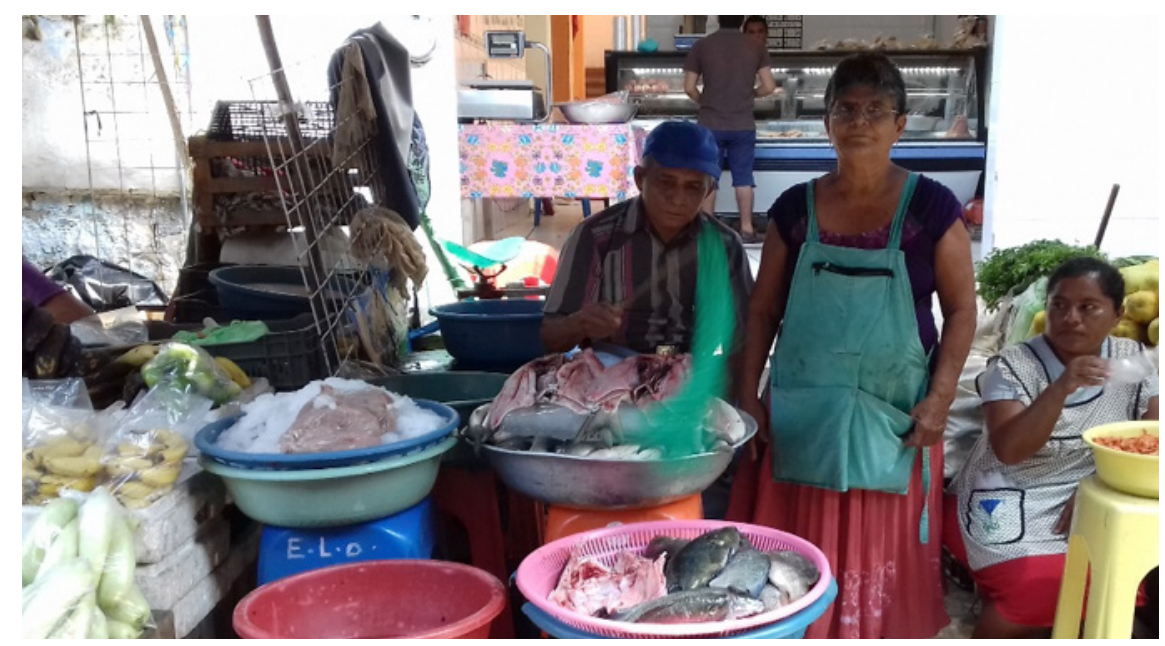

Figura 3. Mujeres rurales vendedoras de pescado en el mercado público Manuel Larrainzar en Tonalá, Chiapas. 


\subsection{SITUACIÓN DE LAS MUJERES RURALES EN CHIAPAS (MÉXICO)}

En 2010, la población que vivía en localidades rurales (menos de 2,500 habitantes) sumaba 26 millones y representaba 23.2 por ciento de la población total del país; alrededor de 13.4 millones son mujeres y 12.9 millones son hombres. La edad mediana de las mujeres rurales es de 23 años y 22 para los hombres, mientras que la correspondiente a las mujeres de grandes ciudades de 100 mil y más habitantes es 29 años, 2 más que los hombres cuya edad mediana es de 27 años (CEAMEG con base en INEGI Estadísticas históricas de México 2009).

En este mismo año, CEAMEG menciona, que había 102 mujeres rurales por cada 100 hombres rurales. Oaxaca es la entidad federativa que tiene una mayor proporción de mujeres rurales $(52.3 \%)$, seguida de Chiapas con la mitad de su población de mujeres residentes en áreas rurales. Hidalgo y Tabasco también tienen una importante proporción de mujeres rurales, 47.4 y 41.9 por ciento, respectivamente.

\section{Las mujeres rurales representan el 504\% del total de mujeres mexicanas.}

Por lo tanto, las mujeres en Chiapas, no se pueden excluir de la situación específica de las mujeres rurales, las cuales representan el 50,4\% del total de las mujeres mexicanas (CEAMEG, 2014). La mayoría de este colectivo, están en situación de pobreza extrema. Además de vivir en comunidades rurales, con una inadecuada infraestructura de servicios, escasos servicios de salud o educativos, y con necesidades básicas insatisfechas en la mayoría de los hogares, viven dominadas por el sistema "machista" propia de una cultura rural, sin reconocimiento de sus derechos, ni de sus labores dentro y fuera del hogar.

Esto limita su integración y participación en actividades comunitarias, o llegar a ser parte de organizaciones donde se toman decisiones relacionadas con el desarrollo. Según Mareswara (2016), las responsabilidades asumidas en función de su sexo (cuidado de la familia, tareas domésticas, cuidado de animales de traspatio, etc.) y otras referidas a las tareas agrícolas reducen sus posibilidades de tener una actividad económica que les genere ingresos para su autonomía, o bien actividades relacionadas con su desarrollo personal que le permita elevar su autoestima.

Deben sobrevivir en un sistema que les ha negado el acceso a recursos productivos, asistencia técnica, 
conocimientos y formas de organización propias. Conscientes de este problema, las instituciones públicas del país han comenzado a abordar el problema y promover los derechos de las mujeres a nivel legislativo y estratégico (CEAMEG, 2014).

Para ello se han elaborado documentos básicos en materia de derechos humanos de las mujeres como: la "Convención sobre la eliminación de todas las formas de discriminación contra la Mujer", aprobada por la ONU en 1979 a nivel internacional, y a nivel regional, la "Convención interamericana para prevenir, sancionar y erradicar la violencia contra la mujer" conocida como Convención de Belém de Pará desde 1999; a nivel nacional, el Congreso de la Unión aprobó en febrero del 2007 la "Ley General de acceso de las mujeres a una vida libre de violencia".

En términos generales se puede decir que el país cuenta con un amplio marco jurídico que protege algunos de los derechos básicos de las mujeres (ver Cuadro No. 1: Derechos Humanos de las mujeres). Sin embargo, aún queda mucho trabajo por realizar para romper con el estigma de la exclusión que pesa sobre las mujeres, principalmente rurales, y que no las reconocen como parte fundamental del desarrollo del país, mucho menos del estado, municipio o comunidad.

Tabla 1. Derechos Humanos de las Mujeres.

\begin{tabular}{|l|c|}
\hline \multicolumn{1}{|c|}{ ACCIÓN } & AÑO \\
\hline Se crea la Comisión de Equidad y Género en la Cámara de Diputados & 1999 \\
\hline Se crea la Ley del Instituto Nacional de las Mujeres & 2001 \\
\hline Se promulga la Ley Federal para Prevenir y Eliminar la Discriminación & 2003 \\
\hline $\begin{array}{l}\text { Se promulga la Ley General para la Igualdad entre Mujeres y Hombres } \\
\text { Se promulga la Ley General de Acceso de las Mujeres a una vida Libre de Violencia. }\end{array}$ & 2006 \\
\hline $\begin{array}{l}\text { Se promulga la Ley para Prevenir y Sancionar la Trata de Personas. } \\
\text { Se tipifica el feminicidio en el Código Penal Federal. }\end{array}$ & 2007 \\
\hline $\begin{array}{l}\text { Ley General de Instituciones y Procedimientos Electorales, la obligación para los partidos políticos de asegurar la } \\
\text { igualdad de oportunidades y la paridad entre hombres y mujeres para tener acceso a cargos de elección popular. }\end{array}$ & 2007 \\
\hline Ley General sobre los Derechos de Niñas, Niños y Adolescentes. & 2012 \\
\hline
\end{tabular}

Fuente: CEAMEG a partir de la Cámara de Diputados 2016. 


\subsection{JUSTIFICACIÓN}

La idea de promover el empoderamiento de las mujeres constituye parte de un cambio cultural, social y económico, entendido como un proceso orientado de forma consciente a transformar ideas, creencias y conductas que afectan la calidad de vida de hombres y mujeres; incluso, el desarrollo de las comunidades rurales (Villarreal, 2011).

Para que la voluntad de modificar el sometimiento de género por el empoderamiento sea efectivo, se debe involucrar a las partes para percibir si desean cambiar y como se debe participar en ese proceso de cambio que se pretende lograr (Narasimha, Anand, Ravish, Navya y Ranganath, 2016). Si no existe el deseo de colaboración entre este colectivo (hombres y mujeres), difícilmente se conseguirá este cambio esperado. Para ello, fue necesario analizar sus costumbres, usos, prácticas, tradiciones e ideas que prevalece en las comunidades rurales con respecto al rol de género.

Por tal motivo, el empoderamiento reconoce la necesidad de aumentar el poder de las mujeres, definido en términos de liderazgo que les permitirá influir en el cambio cultural, económico, político y social a través del control de recursos materiales y no materiales (Hidalgo, 1999).

Pérez, Vázquez \& Zapata (2008) explican que "Género en Desarrollo", considera a las mujeres como sujetos dinámicos y promotores de cambios, por lo que, propone el empoderamiento femenino, como estrategia para superar las limitaciones de lo mencionado ‘por las "Mujeres en Desarrollo". Para Young (1997), el empoderamiento consiste en facilitar el control de las mujeres sobre los procesos que afectan sus vidas, lo que les permitirá vigilar sus propias actividades, organizarse para ayudarse unas a otras y generar peticiones de apoyo a las instancias locales, municipales, estatales y nacionales para incidir en el cambio, desarrollo de la sociedad, del país y del mundo.

La perspectiva de Género en Desarrollo, se ocupa de las relaciones de género en situaciones culturales e históricas concretas. Incluye, en la agenda para el cambio, las necesidades estratégicas de las mujeres, buscando modificar relaciones de desigualdad (Nazar \& Zapata, 2000).

Pérez et al., (2008) continúa comentando las afirmaciones de Género en Desarrollo y analizan el papel de éste colectivo en el ámbito productivo, con el fin de que la política pública no genere nuevas cargas de trabajo para ellas. En su propuesta introduce dimensiones cualitativas, quizá no medibles, del desarrollo, como por ejemplo la importancia de la autonomía femenina, de su independencia económica, de su desarrollo emocional y profesional, así como la necesidad de promover una 
democracia participativa donde la brecha entre líderes e instituciones sea eliminada para que las personas sean sujetos modificadores de sus propios entornos (Pérez, 2001).

Según Rowlands (1997), a partir de la investigación que hizo en grupos de mujeres rurales, el empoderamiento de estas es "un núcleo de procesos psicológicos que cuando se desarrollan, capacitan al individuo o al grupo para actuar de tal forma que incremente su acceso al poder y su uso en varias formas". Eso significa la capacidad para elevar su conciencia como mujeres y el desarrollo de habilidades para superar problemas y encontrar salidas y soluciones.

Villarreal, (2011), comenta del estudio anterior, parecería, “con los datos registrados sobre el número de mujeres que están participando en los mercados públicos, que las mujeres están encontrando caminos para incrementar su participación en las ventas de manera autónoma", lo que sería una pista sobre su avance en términos de empoderamiento. 


\section{METODOLOGÍA}

El diseño de la investigación es de tipo cualitativo y descriptivo, se desarrolló en dos fases para que se capturaran los distintos significados de este fenómeno social y personajes involucrados en este proceso; el problema a estudiar fue el binomio colaboración empoderamiento femenino de las vendedoras de pescado en la periferia del mercado público Manuel Larrainzar en Tonalá, Chiapas. En la primera fase, se llevó a cabo por medio de la observación y el análisis de los testimonios recogidos con el grupo de enfoque realizado, se fue construyendo su significado de acuerdo a los comentarios, opiniones, ideas vivencias y sentimiento de este colectivo (ver Cuadro No. 2: Censo de mujeres vendedoras de pescado).

La información recogida con esta metodología, fue llevada a cabo durante los meses de junio a diciembre del año 2016. A partir de la identificación de las dificultades se construyó una matriz FODA que se utilizó para visualizar una síntesis del binomio colaboración-empoderamiento por parte de las mujeres rurales emprendedoras; iniciando de forma individual y concluyendo de manera colectiva (Tabla 3 ).

Tabla 2. Censo de mujeres vendedoras de pescado.

\begin{tabular}{|l|l|l|l|}
\hline \multicolumn{1}{|c|}{ NOMBRES } & \multicolumn{1}{c|}{ EDAD } & \multicolumn{1}{c|}{ EDO. CIVIL } & HIJOS \\
\hline Nicolasa Trinidad Castañeda & 42 & Casada & 6 \\
\hline Margarita Trinidad Castañeda & 49 & Casada & 8 \\
\hline Teresa de Jesús Matías Trinidad & 30 & Casada & 4 \\
\hline Guadalupe Salazar Mendoza & 40 & Divorciada & 3 \\
\hline Danny Gricel Natarán Roblero & 23 & Soltera & 0 \\
\hline Rosa Alba Lorenzana Ramos & 40 & Casada & 3 \\
\hline Gloria Trujillo & 41 & Viuda & 5 \\
\hline Sandra luz Cigarroa Flores & 38 & Casada & 1 \\
\hline Elidía López Ovando & 67 & Casada & 6 \\
\hline Elio Cueto De los Santos & 68 & Casado & 6 \\
\hline Margarito Matías Mateo & 52 & Casado & 8 \\
\hline Violeta López Caballero & 45 & Casada & 3 \\
\hline Leydi Ovando Ríos & 30 & Casada & 1 \\
\hline María angélica De Paz Trinidad & 23 & Soltera & 0 \\
\hline Graciela De Paz Trinidad & 22 & Soltera & 0 \\
\hline Mari Celia Matías Trinidad & 32 & Madre soltera & 1 \\
\hline Aloína Ovando Antonio & 55 & Unión Libre & 5 \\
\hline
\end{tabular}

Fuente: elaboración propia. 
Tabla 3. Binomio Colaboración-Empoderamiento.

\begin{tabular}{|c|c|c|}
\hline & FORTALEZAS & DEBILIDADES \\
\hline+ & $\begin{array}{l}\text { - Incorporación cada día más a la Educación } \\
\text { - Buscan alternativas de independencia } \\
\text { económica } \\
\text { - Mejoran cada día más su autoestima } \\
\text { - Valoran más su capacidad creativa } \\
\text { - Potencian su desarrollo personal } \\
\text { - Brindan más atención en aspectos de su } \\
\text { salud } \\
\text { - Apoyo mutuo para superarse } \\
\text { - Más información sobre la equidad de } \\
\text { género }\end{array}$ & $\begin{array}{l}\text { - Ausencia de capacitación en nuevas tecnologías } \\
\text { - Bajos conocimientos empresariales } \\
\text { - Falta de organización en los grupos de mujeres } \\
\text { - Carencia de autonomía en la Toma de Decisiones } \\
\text { - Escasez de herramientas de Mercadotecnia. } \\
\text { - Tienen que solicitar autorización para desplazarse } \\
\text { de un sitio a otro, } \\
\text { - Nulos conocimientos contables-administrativos } \\
\text { - Bajo nivel de estudios }\end{array}$ \\
\hline+ & $\begin{array}{l}\text { - Mejorar su calidad de vida } \\
\text { - Poseen mayor voluntad para emprender } \\
\text { - Desafían las leyes de subordinación } \\
\text { - Mayor capacidad de reacción ante los } \\
\text { problemas } \\
\text { - Han ido venciendo el miedo al cambio } \\
\text { - Se han decido a ser protagonistas de su } \\
\text { vida } \\
\text { - Son estupendas negociadoras }\end{array}$ & $\begin{array}{l}\text { - Opresión de parte de los hombres de su entorno } \\
\text { - Escasez de trato afectuoso por parte de sus } \\
\text { padres } \\
\text { - Falta de respeto en el hogar } \\
\text { - Ausencia de involucramiento de los esposos en } \\
\text { labores domésticas. } \\
\text { - Trato desigual hombre/mujer en el espacio familiar. } \\
\text { - Violencia verbal y maltrato familiar }\end{array}$ \\
\hline & OPORTUNIDADES & AMENAZAS \\
\hline
\end{tabular}

Fuente: elaboración propia.

Las mujeres asistentes, que se atrevieron a verbalizar las condiciones de inequidad, opinaron que con la venta de pescado en la periferia del mercado público, existe la posibilidad de mejorar su condición o al menos atenuar su impacto.

Una de ellas decía: "en la comercialización, veo otra perspectiva de vida, me da otra visión". En el grupo, se manifestó preocupación por la mejor manera de introducir cambios y de promover la solidaridad entre mujeres. Esta idea que se relaciona con las actoras sobre las "buenas costumbres" entre las mujeres, facilitarían los procesos organizativos para que pudieran darse apoyo mutuamente que beneficiaría su unidad, facilitando la transición a empoderarse.

También explicaron, que en las actividades de comercialización, que comprende la compra/ venta, es donde se produce la mayor utilidad, pues al realizarse la venta se da la valorización de la mercancía, es decir, se materializa el esfuerzo del trabajo en dinero, y se pueden calcular y comparar los costos de adquisición del producto, así como del valor agregado (ganancia). 


\section{RESULTADOS}

El análisis realizado reveló que de las 17 personas que comercializan su producto en este sitio, 15 son mujeres, y únicamente 2 hombres; de ellas 10 son casadas (os), 3 solteras, 1 que vive en unión libre, 1 madre soltera, 1 divorciada y 1 viuda, como se puede observar el rol protagónico de la mujer en esta actividad es predominante.

Estas mujeres tienen como apoyo económico ahorros propios, tandas, préstamo que solicitan a familiares y amigos, porque los sistemas crediticios o microcréditos les cobran demasiados intereses propiciando que no lo puedan pagar.

Con respecto a los ingresos que reciben por las ventas que realizan, se reflejó que las mujeres asumen más responsabilidades y compromisos que los hombres, puesto que después de haber cumplido con el total de sus ventas, el ingreso que obtienen de su negocio, un porcentaje es para comprar su mercancía al día siguiente; otra porción, para el pago de sus pasajes y traslados al lugar donde realizan su compra e ir al lugar donde está su expendio, incluso tienen que separar otro porcentaje para enviar a sus hijos a la escuela, otra fracción para el alimento diario de su familia.

Estas mujeres tienen como apoyo económico ahorros propios, tandas, préstamo que solicitan a familiares y amigos, porque los sistemas crediticios o microcréditos les cobran demasiados intereses propiciando que no lo puedan pagar. También reciben apoyos económicos estatales, cuya cantidad oscila entre 2 mil a 3 mil pesos como beca a sus hijos que están en la escuela. Esto les da un fuerte impulso económico, lo que les permite "refaccionarse" y tener efectivo para comprar su producto (pescado).

Habitualmente este negocio no necesita que las personas tengan gran experiencia en el trabajo, es suficiente que sepan "realar" el pescado, es decir, limpiarlos y abrirlos; preparándolos para darles la presentación que solicita el cliente, de acuerdo a como lo requieran en su hogar para su alimento (frito, en caldo, empanizado, al horno, asado, ceviche, al hervor, al agua chile, tapadito, empapelado etc.,). La forma de "comerciar" la van adquiriendo conforme pasa el tiempo y venden su mercancía (Pérez et al. 2014)

La edad de las vendedoras de pescado, fluctúa en un 60 por ciento de 30 a 50 años, otro porcentaje menor el 23 por ciento son mayores de 50 años y únicamente el 17 por ciento corresponden de 20 
a 29 años. En cuando a la edad de cuando iniciaron este negocio, la mitad de ellas comenzaron este comercio a la edad de 20 a 40 años y la otra mitad de las propietarias comenzaron este negocio a una edad de 20 años y menores de 20.

Al decir de ellas, la mayoría tuvo algo de planeación no escrita cuando empezó su negocio y solo la minoría hizo un plan de negocios, hay que aclarar cuando ellas hablan de planeación, es cuando van a preguntarle a sus familiares o amigos, acerca de poner un pequeño negocio, incluso, se llega al caso, que van y hablan con los dirigentes de sus sectas (las cuales hay muchas en estos lugares y de las que son integrantes). Este mismo caso pasa cuando hablamos de asesoría profesional, aunque la mayoría dijo que no tuvo asesoría profesional, se da mucho el caso anterior (Pérez et al. 2014).

Por lo tanto, se pudo observar que la educación es uno de los factores principales que afectan a la labor que realizan; otro factor no menos importante es el tiempo que le dedican a sus actividades comerciales, a menor tiempo, menor espacio empleado para su trabajo. Otro factor, son las razones personales y/o familiares, que las limita, tomando la preferencia de no ir más allá en su comercio. Generalmente estas propietarias tienen un nivel educativo máximo de primaria, algunas de ellas tienen secundaria y solo un grupo minoritario tienen preparatoria, actualmente dos de ella están estudiando licenciatura en Contaduría Pública lo que potenciará su conocimiento para llevar el registro de sus gastos y costos en su negocio. 


\section{CONCLUSIONES Y PROPUESTA DE MEJORA}

Poner fin a la pobreza en todas sus formas, también tiene una resonancia especial para las mujeres rurales. La interrelación entre el acceso limitado a los recursos, la educación y la atención médica inapropiadas, la discriminación sistemática y los obstáculos a su participación agravan su condición de pobreza y exclusión.

Por ello, el empoderamiento de las mujeres rurales, es un requisito previo para lograr la visión de los Objetivos de Desarrollo Sostenible 2030, que se propone "poner fin a la pobreza y el hambre, alcanzar la seguridad alimentaria y empoderar a todas las mujeres y las niñas como agentes clave en los sistemas alimentarios", tan prioritario para este colectivo que se encarga de garantizar la alimentación adecuada para las familias; las mujeres rurales se encuentran en el centro de este desafío.

Si se logra que el grupo de mujeres rurales emprendedoras que venden pescado en la periferia del mercado público Manuel Larrainzar se organicen para acceder a créditos, apoyos municipales, estatales y nacionales, además, se les proporcione una alternativa de distribución, así como dotarlas de herramientas estratégicas de mercadotecnia para que mejoren la comercialización, imagen e higiene de su producto, solo entonces se podrá garantizar que ellas alcanzarán su nivel de bienestar mínimo para satisfacer las necesidades económicas de su familia, hijos, padres, abuelos y todos aquellos que dependen de este colectivo.

El empoderamiento de las mujeres rurales, es un requisito previo para lograr la visión de los Objetivos de Desarrollo Sostenible 2030, que se propone "poner fin a la pobreza y el hambre, alcanzar la seguridad alimentaria y empoderar a todas las mujeres y las niñas como agentes clave en los sistemas alimentarios".

Finalmente, se debe involucrar a todos los integrantes de la familia a que participen activamente en las labores diarias del hogar, para propiciar que las mujeres descarguen sus quehaceres y responsabilidades, que les permita tener un horario de esparcimiento y relax para su bienestar emocional que repercutirá en el progreso de todos los miembros de la familia y ellas puedan lograr su pleno desarrollo personal y social que detone su mejoría económica y la de su entorno. 


\section{AGRADECIMIENTOS}

- Al COCYTECH por el apoyo económico proporcionado para la realización de este estudio.

- A las mujeres rurales emprendedoras que venden pescado en la periferia del Mercado público Manuel Larrainzar en Tonalá, Chiapas. "gracias por su participación".

\section{REFERENCIAS BIBLIOGRÁFICAS}

Centro de Estudios para el adelanto de las Mujeres y la Equidad de Género (GEAMEG). (2016). Las reformas a la Constitución de 1917 desde el punto de vista de las mujeres. Cámara de Diputados LXIII Legislatura. México, D.F.

García Canclini, N. (2003). Culturas hibridas. Estrategias para entrar y salir de la modernidad. Editorial Grijalbo, México, D. F.

Hidalgo, C.N. (1999). Cajas de ahorro como estrategia de sobrevivencia de mujeres rurales: Caso de la organización SSS Susana Sawyer, Álamos, Sonora. Tesis de Maestría en Estudios del Desarrollo Rural, México, Colegio de Postgraduados.

La Agenda 2030 y los Objetivos para el Desarrollo Sostenible. (2015). Agenda 2030 y los Objetivos de Desarrollo Sostenible. Una oportunidad para América Latina y el Caribe. CEPAL. ONU.

Mareswara Rao, N. (2016). A Study on Empowerment of Rural Women Through Entrepreneurial Activities. Social Science. 5(10), 311-313.

Narasimha B.G., et al. (2016). Role of self help groups in women empowerment and health. International Fournal of Community Medicine and Public Health, 3(8), 2026-2028.

Nazar, A. \& Zapata, E. (2000). Desarrollo bienestar y género: consideraciones teóricas. La Ventana, 11, 73-118.

Pérez, N.E. (2001). El proceso de empoderamiento de mujeres indígenas organizadas desde una perspectiva de género. Estudios Agrarios, 17, 125-169. 
Pérez, P.I.; Orozco, G.M. y Cruz, S.Z.M. (2014). Mujeres rurales emprendedoras de la región Istmo-Costa de Chiapas: productoras de totopo y memelita de olla. Researchetnet, 184-201.

Pérez V.M., Vázquez, G.V. y Zapata M.E. (2008). Empoderamiento de las mujeres indígenas de Tabasco. El papel de los fondos regionales de la CDI. Colegio de Postgraduados, Campus Montecillo, Texcoco, Estado de México. Revista Cuicuilco, 15(42).

Rodríguez, B.E. (2009). Género, Cultura y Desarrollo: Límites y oportunidades para el cambio cultural pro-igualdad de género en Mozambique. Instituto Complutense de Estudios Internacionales, Universidad Complutense de Madrid. Madrid, España.

Young, K. (1997). El potencial transformador en las necesidades prácticas: empoderamiento colectivo y el proceso de planificación, en León, M. (Ed.), Poder y empoderamiento de las mujeres, Bogotá, Tercer Mundo, 99-118.

Villarreal, M. (2011). Mujeres rurales y oportunidades: de la participación al empoderamiento. Tend-Red, 16, 243-259. Colombia. 